\title{
Overtime, Shift Work, Poor Sleep and the Effects on Obesity: A Public Health Problem
}

\section{Pouran D Faghri* and Jennifer Buden}

Department of Allied Health Sciences-Health Promotion, University of Connecticut, USA

*Corresponding author: Dr. Pouran D Faghri, Department of Allied Health Sciences-Health Promotion, University of Connecticut, USA, Tel: 860-486-0018; E-mail: pouran.faghri@uconn.edu

Rec date: May 19, 2016; Acc date: May 20, 2016; Pub date: May 26, 2016

Copyright: (c) 2016 Faghri PD et al. This is an open-access article distributed under the terms of the Creative Commons Attribution License, which permits unrestricted use, distribution, and reproduction in any medium, provided the original author and source are credited.

\section{Editorial}

Over two-thirds $(68.6 \%)$ of adults in the United States were overweight or obese in 2011-2012, and prevalence rates continue to increase at a dramatic pace [1]. A recent review of the literature revealed that direct medical costs of obesity and comorbidities such as cardiovascular disease and type 2 diabetes may be as high as $\$ 209.7$ billion annually [2]. Indirect costs may sum near $\$ 66$ million annually, with potential to yield significant savings to individuals, employers, and improve quality of life [2]. Understanding potential factors to reduce and prevent further increases in the obesity epidemic has the potential to yield over $\$ 500$ billion dollars in savings by the year 2030 [2]. Analysis of work-related factors such as worksite health culture, overtime, shift work and lifestyle behaviors influenced by the workplace may be one approach to address the obesity epidemic.

According to the Bureau of Labor Statistics, approximately 122 million Americans work full-time [3]. With consideration that Americans spend over one-third of their time at work, the workplace is in a unique position to utilize health promoting practices [4]. Efforts to employ a healthy workforce by fostering a healthy environment and providing policies, programs, and resources supportive of health has the potential to produce significant economic benefit. In addition, strategies to address the obesity epidemic will require significant restructuring of work conditions and policies, specifically in high stress occupations with unstructured overtime and shift work. The purpose of this paper is to examine the associations among work behaviors such as overtime and shift work, lifestyle behaviors (nutrition, physical activity, sleep) and obesity in high-stress occupations.

The National Sleep Foundation recommends adults get 7-9 hours of sleep per night [5]. Inadequate sleep is recognized as a well-known independent risk factor to obesity and other comorbid conditions such as diabetes and cardiovascular diseases. Researchers increasingly consider lack of sleep a public health issue, as it is associated with changes in glucose metabolism [6], immune response [7], weight gain [8-10], and psychological well-being [11]. Beyond intrapersonal outcomes, poor sleep may have a negative impact on employers due to its' association with losses in productivity and poor safety outcomes [12]. A study by Rosekind et al. [12] of over 4000 employees working in the United States selected from diverse industries and geographic regions reported that the economic impact of lost productivity due to poor sleep from this cohort alone would approximate to $\$ 54$ million annually or $\$ 1967$ per employee [12]. In an effort to address the obesity epidemic, burden on workplace productivity, and health-related quality of life of employees, it is important to understand contributing risk factors to inadequate sleep quantity and quality, such as overtime shift work.
Overtime and long hours of shift work may provide financial benefits to employees seeking increased income and for employers who wish to avoid the hiring process. However, a meta-analysis by Sparks et al. [13] examining the effects of increased working hours on health found that working more hours was associated with higher physiological and psychological health symptoms, with potential mediating and moderating factors [13]. Further, increased overtime work has been associated with poor health [14], higher prevalence of injury rates [15], cardiovascular events [16], musculoskeletal disorders [17] and unhealthy behaviours $[14,18,19]$. Furthermore, long shifts are associated with poor performance, decreased attention, productivity and increased injuries [14].

According to the National Health Interview Survey (NHIS, 2004-2008), increased time spent at work and poor sleep are both independently and significantly associated with elevated rates of work injury, even when controlling for potential confounding and demographic variables, such as occupation. This finding is significant in that increased working hours alone, and poor sleep alone may increase work injury, and therefore the combination of the two is likely even more problematic [20]. When examining worksite policies, understanding how occupation-specific factors may impact the relationship between overtime and health outcomes requires future research. Factors such as number of rest days, length of work week, rotation of shifts, worksite culture, and control in the workday are unique occupational factors that may indirectly influence the impact of overtime on health [14].

Typically, individuals spending more time in the workplace have less time available for leisure activities, sleep, and other health-promoting behaviours that influence long-term health status such as, overweight, obesity and chronic disease risk. The costs associated with poor sleep, unhealthy lifestyle behaviours and obesity extend beyond the worksite organization and negatively affect the individual and society. Numerous studies have reported on negative changes in sleep quality in employees working rotating or night shifts [21-23]. Further, employees working night shift may exhibit differences in lifestyle habits, such as increased sugar-sweetened beverage consumption [22], higher caloric intake and smoking status [23] which in turn is associated with increased BMI, $[22,24,25]$ and elevated chronic disease risk [23].

One study by Taris et al. [26] examined the relationships between overtime, health behaviours and health outcomes to determine the mechanism behind these factors. The authors reported that higher overtime was associated with less health protective behaviours and lower subjective health. Further, they found that health behaviours did not fully explain this relationship, indicating that there are potentially other factors such as psychological and physiological factors playing a 
role. Authors' direct future research efforts towards examination of health behaviours, in particular health protective behaviours that may be moderated and altered by overtime and stress. It is likely that nutrition, physical activity and sleep are the primary behaviours negatively influenced by excess hours spent at work because they require the most conscious effort by an individual. These behaviours should be the target of future health promotion efforts [26].

Occupational characteristics such as rotating shifts, job strain, and uncontrollable stressors in the workday may increase risk for many chronic diseases including obesity. For example, a study by Morse et al. [27] reporting on correctional officer health presented numerous health barriers related to overtime and sleep patterns. Over $80 \%$ of participants were overweight or obese and there was a significant association between shift (first, second or third) and BMI $(\mathrm{p}<0.01)$. Over half of the participants in the study (52\%) reported sleeping 6 or less hours per night on average. Overtime work was cited as a barrier to fitting in fitness and healthy eating [27].

A cross-sectional study by Ramey et al. [28] on male police officers reported that officers working night shift were 14 times more likely to sleep 6 hours or less per day $(\mathrm{p}<0.001)$, with differences in sleep quality. In addition, those with less sleep had a higher percentage of being overweight or obese (88\%) compared to those with more sleep (78\%), though this did not reach statistical significance. Respondents in this study working day shift reported frequent overtime (53\%) and the average work week was 46 hours. Those working non-day shift were more likely to work shifts back-to-back, which may increase job exhaustion, burnout, and sleep deprivation [28].

Numerous studies provide strong evidence of an association between inadequate sleep and obesity risk [8,29-31]. Further clarifying these findings, one meta-analysis concluded that this relationship is not evident for adults who are getting adequate, or longer durations of sleep [30]. Proposed explanations behind the causality of this relationship have been inconclusive. For example, one review article on epidemiological evidence examining short sleep duration and obesity risk questioned the limitations of assuming that there is a causal and linear pathway in which this relationship exists. Other comorbid conditions such as depression, psychosocial issues, age and stress may act as confounding factors in the sleep and obesity relationship. Interactions between external and environmental factors (ex: long work days, commute time) with internal factors may share a complex relationship activating physiological pathways [29]. Other commonly proposed mechanisms attempting to explain this relationship include: changes in eating habits [32], changes in hormone regulation altering serum ghrelin and leptin levels causing increased appetite [33], greater fatigue reducing physical activity levels [34], and physiological changes in inflammatory pathways [35,36] and metabolism [37].

Research to-date has been inconclusive regarding changes in lifestyle behaviours such as increased caloric intake and lack of exercise $[32,34]$. Rather, studies have demonstrated changes in eating choices, such as a greater proportion of caloric intake from beverages and snacks, which may be associated with sleep loss [32]. It is likely that behavioural changes influencing weight status may be dependent on acute versus chronic sleep deprivation. This finding is important for occupations that are organized by rotating shifts, long working shifts, and frequent overtime hours interfering with sleep duration. There is a need for improved methodology and higher level designs to make inferences about causality through understanding of potential comorbidities and mechanisms. This would allow researchers to draw conclusions and propose that modification of sleep patterns would exhibit weight loss or protection against obesity progression [38,39].

One cross-sectional study by Tsuboya et al. [40] investigating coronary heart disease risk and working hours conducted in Japan reported dose-response associations among overtime work with sleep deprivation and psychological stress. The study was unable to report associations with overweight or obesity, but contributes this contradicting finding to the low incidence of these health conditions in the population studied [40].

A study titled the "Shift Length Experiment" by Amendola et al. [41] examined police officers working different shift lengths and the effect on important health and safety outcomes. The researchers found that individuals working 10-hour shifts got more sleep, worked less overtime and had higher rated work quality of life compared to those working 8-hour shifts. The group working 12-hour shifts displayed similar results, however, negative outcomes such as decreased alertness on the job suggest this may not be the best solution for health and safety initiatives. This study demonstrates important findings for use in changing organizational policies in the public safety sector. Both employees and employers may see immediate and long-term benefits from changing shift rotations. This study proposes that 4 days of 10hour shifts may be a cost-effective solution to reduce sleep deprivation and mandated overtime, and improve the health, safety, and quality of life for employees [41].

In conclusion, numerous studies have demonstrated concerning associations among poor sleep and long working hours with elevated risk of obesity and chronic disease. It is vital that future research explores the relationships among sleep, overtime work and obesity in high stress occupations that may exhibit differing effects than the general population. With understanding of the health outcomes associated with poor sleep and excess working hours, development of health promoting policies and initiatives may provide cost savings to employees and employers. Organizational health change may benefit from reducing job strain and burnout by considering innovative shift practices that reduce mandated overtime to decrease fatigue, obesity and injuries in the workplace. Utilizing a Total Worker Health approach, shift assignments such as 4-day, 10-hour shifts or allowance of split overtime shifts may improve health and safety in the workplace.

\section{References}

1. Ogden CL, Carroll MD, Kit BK, Flegal KM (2014) Prevalence of childhood and adult obesity in the United States, 2011-2012. JAMA 311: 806-814.

2. Spieker EA, Pyzocha N (2016) Economic Impact of Obesity. Prim Care 43: 83-95.

3. Bureau of Labor Statistics (2016) Monthly number of full-time employees in the united states from february 2015 to february 2016 (in millions, seasonally adjusted). Statista - The Statistics Portal Web site.

4. United States Department of Labor (2016) American time use survey. Bureau of Labor Statistics Web site.

5. Hirshkowitz M, Whiton K, Albert SM, Alessi C, Bruni O, et al. (2015) National sleep foundation's updated sleep duration recommendations: Final report. Sleep Health: Journal of the National Sleep Foundation 1: 233-243.

6. Spiegel K, Tasali E, Leproult R, Van Cauter E (2009) Effects of poor and short sleep on glucose metabolism and obesity risk. Nat Rev Endocrinol 5: 253-261.

7. Heffner KL, Ng HM, Suhr JA, France CR, Marshall GD, et al. (2012) Sleep disturbance and older adults' inflammatory responses to acute stress. Am J Geriatr Psychiatry 20: 744-752.

8. Patel SR, Hu FB (2008) Short sleep duration and weight gain: a systematic review. Obesity (Silver Spring) 16: 643-653. 
9. Chaput JP, Després JP, Bouchard C, Tremblay A (2008) The association between sleep duration and weight gain in adults: a 6-year prospective study from the Quebec Family Study. Sleep 31: 517-523.

10. Kobayashi D, Takahashi O, Deshpande GA, Shimbo T, Fukui T (2012) Association between weight gain, obesity, and sleep duration: a large-scale 3-year cohort study. Sleep Breath 16: 829-833.

11. Doi Y, Minowa M, Tango T (2003) Impact and correlates of poor sleep quality in Japanese white-collar employees. Sleep 26: 467-471.

12. Rosekind MR, Gregory KB, Mallis MM, Brandt SL, Seal B, et al. (2010) The cost of poor sleep: workplace productivity loss and associated costs. J Occup Environ Med 52: 91-98.

13. Sparks K, Cooper C, Fried Y, Shirom A (1997) The effects of hours of work on health: A meta-analytic review. J Occup Organ Psychol 70: 391-408.

14. Caruso CC, Hitchcock EM, Dick RB, Russo RB, Schmit JM (2004) Overtime and extended work shifts: Recent findings on illnesses, injuries, and health behaviors. U S Department of Health and Human Services: National Institute for Occupational Safety and Health (NIOSH).

15. Dembe AE, Erickson JB, Delbos RG, Banks SM (2005) The impact of overtime and long work hours on occupational injuries and illnesses: New evidence from the united states. Occup Environ Med 62: 588-597.

16. Liu Y, Tanaka H, Fukuoka Heart Study Group (2002) Overtime work, insufficient sleep, and risk of non-fatal acute myocardial infarction in Japanese men. Occup Environ Med 59: 447-451.

17. Bergqvist U, Wolgast E, Nilsson B, Voss M (1995) Musculoskeletal disorders among visual display terminal workers: Individual, ergonomic, and work organizational factors. Ergonomics 38: 763-776.

18. Trinkoff AM, Storr CL (1998) Work schedule characteristics and substance use in nurses. Am J Ind Med 34: 266-271.

19. Kirk MA, Rhodes RE (2011) Occupation correlates of adults' participation in leisure-time physical activity: a systematic review. Am J Prev Med 40: 476-485.

20. Lombardi DA, Folkard S, Willetts JL, Smith GS (2010) Daily sleep, weekly working hours, and risk of work-related injury: US National Health Interview Survey (2004-2008). Chronobiol Int 27: 1013-1030.

21. Huth JJ, Eliades A, Handwork C, Englehart JL, Messenger J (2013) Shift worked, quality of sleep, and elevated body mass index in pediatric nurses. J Pediatr Nurs 28: e64-73.

22. Tada Y, Kawano Y, Maeda I, Yoshizaki T, Sunami A, et al. (2014) Association of body mass index with lifestyle and rotating shift work in japanese female nurses. Obesity (Silver Spring) 22: 2489-2493.

23. Ramin C, Devore EE, Wang W, Pierre-Paul J, Wegrzyn LR, et al. (2015) Night shift work at specific age ranges and chronic disease risk factors. Occup Environ Med 72: 100-107.

24. Antunes Lda C, Jornada MN, Ramalho L, Hidalgo MP (2010) Correlation of shift work and waist circumference, body mass index, chronotype and depressive symptoms. Arq Bras Endocrinol Metabol 54: 652-656.

25. Di Milia L, Mummery K (2009) The association between job related factors, short sleep and obesity. Ind Health 47: 363-368.

26. Taris TW, Ybema JF, Beckers DG, Verheijden MW, Geurts SA, et al. (2011) Investigating the associations among overtime work, health behaviors, and health: A longitudinal study among full-time employees. Int J Behav Med 18: $352-360$.

27. Morse T, Dussetschleger J, Warren N, Cherniack M (2011) Talking about health: Correction employees' assessments of obstacles to healthy living. J Occup Environ Med 53: 1037-1045.

28. Ramey SL, Perkhounkova Y, Moon M, Budde L, Tseng HC, et al. (2012) The effect of work shift and sleep duration on various aspects of police officers' health. Workplace Health Saf 60: 215-222.

29. Nielsen LS, Danielsen KV, Sørensen TI (2011) Short sleep duration as a possible cause of obesity: critical analysis of the epidemiological evidence. Obes Rev 12: 78-92.

30. Wu Y, Zhai L, Zhang D (2014) Sleep duration and obesity among adults: a meta-analysis of prospective studies. Sleep Med 15: 1456-1462.

31. Cappuccio FP, Taggart FM, Kandala NB, Currie A, Peile E, et al. (2008) Meta-analysis of short sleep duration and obesity in children and adults. Sleep 31: 619-626.

32. Kant AK, Graubard BI (2014) Association of self-reported sleep duration with eating behaviors of american adults: NHANES 2005-2010. Am J Clin Nutr 100: 938-947.

33. Taheri S, Lin L, Austin D, Young T, Mignot E (2004) Short sleep duration is associated with reduced leptin, elevated ghrelin, and increased body mass index. PLoS Med 1: e62.

34. Schmid SM, Hallschmid M, Jauch-Chara K, Wims B, Benedict C, et al. (2009) Short-term sleep loss decreases physical activity under free-living conditions but does not increase food intake under time-deprived laboratory conditions in healthy men. Am J Clin Nutr 90: 1476-1482.

35. Miller MA, Cappuccio FP (2007) Inflammation, sleep, obesity and cardiovascular disease. Curr Vasc Pharmacol 5: 93-102.

36. Liu R, Liu X, Zee PC, Hou L, Zeng Z, et al. (2014) Association between sleep quality and C-reactive protein: Results from national health and nutrition examination survey, 2005-2008. PLoS One 9: e92607.

37. McHill AW, Melanson EL, Higgins J, Connick E, Moehlman TM, et al. (2014) Impact of circadian misalignment on energy metabolism during simulated nightshift work. Proc Natl Acad Sci U S A. 111: 17302-17307.

38. Marshall NS, Glozier N, Grunstein RR (2008) Is sleep duration related to obesity? A critical review of the epidemiological evidence. Sleep Med Rev 12: 289-298.

39. Coughlin JW, Smith MT (2014) Sleep, obesity, and weight loss in adults: is there a rationale for providing sleep interventions in the treatment of obesity? Int Rev Psychiatry 26: 177-188.

40. Tsuboya T, Aida J, Osaka K, Kawachi I (2015) Working overtime and risk factors for coronary heart disease: A propensity score analysis based in the J-SHINE (Japanese study of stratification, health, income, and neighborhood) study. Am J Ind Med 58: 229-237.

41. Amendola K, Weisburd D, Hamilton E, Jones G, Slipka M (2011) The shift length experiment: What we know about 8-, 10-, and 12-hour shifts in policing. 